\title{
Los medios estatales de Argentina a partir de la asunción de la alianza Cambiemos en el gobierno nacional (2016-2018)
}

The state media of Argentina since the assumption of the Cambiemos alliance in the national government (2016-2018)

\author{
Alejandro Linares linares.alejandro.felix@gmail.com \\ https://orcid.org/0000-0002-0228-3927 \\ Instituto de Estudios de América Latina y el Caribe; Facultad de Ciencias Sociales; \\ Universidad de Buenos Aires/ Universidad Nacional de La Pampa (Argentina)
}

María Andrea Mallimaci anmallimaci@gmail.com

https://orcid.org/0000-0003-3489-544X

Instituto de Estudios de América Latina y el Caribe; Facultad de Ciencias Sociales;

Universidad de Buenos Aires/ Universidad de Ciencias Empresariales y Sociales /Universidad

Metropolitana para la Educación y el Trabajo

\section{Resumen}

Este trabajo releva el desempeño institucional de los medios del Estado nacional de la Argentina de 2016 a 2018, a partir de la asunción de la alianza Cambiemos en el gobierno nacional. Se analiza las principales decisiones que delinearon una política para su funcionamiento. 
Para esto, se reseñan las modificaciones normativas, las iniciativas concretas y se recuperan las definiciones de los responsables institucionales. El diagnóstico sobre las tendencias y perspectivas en esta política se nutre de documentos públicos, entrevistas periodísticas y personales a sus directores, la normativa específica y textos académicos vinculados a la cuestión.

El trabajo concluye que a partir de la llegada de un nuevo gobierno nacional argentino en el cierre de 2015 se evidencia un cambio de perspectiva en la gestión de los medios estatales nacionales. En esta tendencia, las emisoras estatales se desplazan de un rol complementario y protagonista a otro subsidiario y marginal dentro del sistema mediático.

Palabras clave: Medios estatales; medios públicos; políticas de comunicación; Argentina.

\section{Abstract}

This paper analyzes the institutional performance of the Argentinean's national state media during 2016, 2017 and 2018, from the takeover of the alliance Cambiemos in the national government. It addresses the main decisions that delineated a policy for its operation.

For this, it reviews the normative modifications, the concrete actions and the conceptual definitions of the main institutional managers. The review of the trends and perspectives in this policy is fed by public documents, journalistic and personal interviews with its directors, specific regulations and academic texts related to the issue.

The article concludes that after the arrival of the national government in Argentina at the end of 2015 it takes place a change of perspective in the management of national state media. In this tendency, these media move from a complementary and protagonist role to a subsidiary and marginal role within the media system.

Keywords: State media; public media; communication policy; Argentina.

En la Argentina se registró entre 2003 y 2015 una paulatina, creciente y renovada actividad del Ejecutivo nacional tanto para potenciar las condiciones de los medios estatales existentes como para crear nuevas señales. En ese periodo, que tuvo al kirchnerismo en el Gobierno nacional de la Argentina, se lanzaron siete nuevas señales temáticas dependientes de organismos del Estado nacional, que asumió un rol emisor, productor y financiador de la producción audiovisual (Becerra y Mastrini, 2016).

Esta iniciativa estatal se produjo en un contexto mediático nacional que no modificó sus características salientes como el dominio de la lógica comercial, la concentración en la 
propiedad y el centralismo en la producción de contenidos (Becerra y Mastrini, 2017). Tampoco cambió otro rasgo característico: la alta penetración de la televisión paga. Según la Encuesta Nacional de Consumos Culturales de 2017, 69,3\% de los ciudadanos aseguraba mirar habitualmente la TV con esos servicios por suscripción (Sinca, 2018).

Este panorama en las experiencias audiovisuales generadas desde el Estado nacional significaba todo un desafío para el Gobierno que asumió en diciembre de 2015. Con casi tres años transcurridos de gestión en el Ejecutivo se estabilizaron algunas tendencias en las políticas dirigidas al sector audiovisual en general, y referidas a los medios del Estado nacional en particular. Esto abre un campo de interrogación relevante para alcanzar balances y proponer miradas prospectivas.

El ascenso de una alianza partidaria alternativa a la fuerza política que gobernó en los trece años previos al periodo de análisis ofrece una oportunidad para interrogar sobre continuidades y rupturas en la administración de los medios que dependen del Ejecutivo nacional.

\section{Propuesta metodológica}

El trabajo enfoca su análisis en las decisiones sobre los diferentes medios de comunicación a cargo del Estado nacional, que a partir del 11 de diciembre de 2015 pasaron a estar englobados institucionalmente por el Sistema Federal de Medios y Contenidos Públicos (en adelante SFMCP), entidad con rango ministerial creada el primer día del Gobierno de la fuerza Cambiemos. Se entiende entonces al SFMCP como principal referente empírico del análisis, aunque en algunos apartados se focaliza particularmente sobre Radio y Televisión Argentina Sociedad del Estado (en adelante, RTA), principal empresa estatal encargada de los medios del Estado nacional.

El periodo abordado se extiende desde 11 de diciembre de 2015 al 30 de septiembre de 2018 , que comprende los primeros dos años y nueve meses de gestión de la alianza partidaria Cambiemos. Esta etapa permite reconocer las tendencias evidenciadas en los medios analizados en el marco de un nuevo periodo político.

El objetivo del artículo es reconstruir los contornos de las políticas de comunicación que afectaron el funcionamiento de los medios administrados por el Estado nacional. Para esto, se reseñan las modificaciones normativas y las iniciativas desplegadas para el funcionamiento institucional, la producción de contenidos y el financiamiento. El trabajo reconoce tendencias en esas políticas y quiebres respecto del periodo previo. 


\section{Marco teórico: las políticas de comunicación}

Este trabajo retoma a Oszlak y O'Donnell (1984) en su definición sobre una política estatal, que es entendida como un conjunto de iniciativas y respuestas que pueden ser manifiestas o implícitas, y que permite inferir una cierta posición predominante, pero no absoluta ni definitiva, del Estado frente a una cuestión. Los autores reconocen que estas iniciativas y respuestas no necesariamente son homogéneas y coherentes, sino que pueden ser inconsistentes y conflictivas. En definitiva, se resalta "el carácter negociado o abiertamente conflictivo que frecuentemente asumen las tomas de posición del Estado frente a una cuestión" (1984: 566). Desde este acercamiento, este trabajo entiende que el modo de administrar los medios del Estado forma parte de una política de comunicación, definida como un "conjunto de normas construidas en base a una serie de principios que garanticen que las políticas serán democráticas". Con esto no se refiere sólo a legislaciones "sino a una dimensión material y simbólica que excede el ámbito jurídico y se articula en una disputa de poder entre los diferentes actores sociales" (Monje, 2006: 4). Se intenta incluir dentro de la política de comunicación no sólo a las acciones y mecanismos formales -que supone la legislación, el proceso de su gestación, los instrumentos y modos de su aplicación- sino también a las instancias informales que inciden tanto en la elaboración como en la aplicación de una política pública.

Existen políticas impulsadas concretamente para incidir en el funcionamiento de los medios estatales y otras que tienen impacto sobre ellos aunque no los reconozcan como destinatarios principales. En ambos casos se trata de decisiones, medidas o regulación específica que forman parte de una política de comunicación considerada en términos globales. De esta última se pueden trazar definiciones o balances según un particular periodo político o temporal retomado.

\section{Medios públicos, libertad de expresión y derecho a la comunicación}

Expresiones como "medios públicos" o "emisoras de servicio público" apuntan a resaltar la búsqueda de objetivos sociales y culturales, en contraposición a objetivos de rentabilidad económica. Tributarias de las históricas experiencias europeas de servicio público de radiodifusión, con estas denominaciones se intenta remarcar un conjunto de principios y requerimientos que esos medios deben cubrir y que los diferencian de emisoras privadacomerciales o comunitarias. Con esta mención se conserva la capacidad de referenciar 
mandatos sociales, una tradición de gestión pública y los principios democráticos a los que debe atender.

Pasquali (1991) y Fuenzalida (2001) han enumerado estas guías rectoras para la construcción de medios públicos en América Latina. Desde estos aportes es posible señalar algunos mandatos para la construcción de medios públicos, como por ejemplo: universalidad, continuidad y renovación tecnológica; pluralismo y diversidad; participación social en las decisiones y producciones; dirección política colegiada diferenciada de la gestión ejecutiva; abordaje crítico de las presiones de diversos sectores en las decisiones del directorio; equidistancia editorial respecto a interferencias gubernamentales, de otros poderes del Estado, partidarias o de actores comerciales; financiamiento público adecuado y estable en el tiempo.

La preocupación central en el análisis de los llamados medios públicos desde una perspectiva de la libertad de expresión debe apuntar al modo en que asumen los mandatos reseñados en su actividad y al modo en que se insertan en un proyecto democratizador de las comunicaciones.

Al mismo tiempo, la perspectiva del derecho a la comunicación vinculada a los medios estatales se relaciona con el modo en que el Estado acciona para garantizar mayores niveles de libertad y pluralidad a la ciudadanía; esto es, funcionando como garante del derecho a la comunicación. Así, los medios públicos se entienden como elementos fundamentales para garantizar contenido inclusivo, federal, destinado a las minorías, por fuera del criterio de rentabilidad y enmarcado en la lógica de la función social. La propia definición del Estado como garante del derecho humano a la comunicación, implica que el mismo no sea una mera declamación, sino que precisa la acción afirmativa del Estado para garantizar su plena vigencia (Loreti y Lozano, 2015).

La responsabilidad de los medios públicos en un escenario mediático concentrado y comercial (Becerra, 2015) aparece fuertemente ligada al derecho a la comunicación, ya que concentración e interés público no pueden ser disociados y la concentración de la propiedad tiene como correlato la homogeneización de contenidos (Loreti y Lozano, 2014: 143). La tarea de los medios públicos, entonces, en relación con la libertad de expresión y el derecho a la comunicación es crucial para garantizar la plena vigencia de la ciudadanía en tanto ejercicio ciudadano de las personas con la esfera pública.

En este trabajo se prefiere utilizar mayormente la denominación medios estatales o del Estado, que reconoce a tales medios por su propiedad o administración estatal, sin desconocer los mandatos y responsabilidades que deben asumir en tanto medios públicos. 


\section{Cambios normativos para una nueva política}

En el inicio del periodo del gobierno nacional de la fuerza política Cambiemos, el Estado tenía la propiedad de un conjunto de medios de comunicación que funcionaba en la órbita del Ejecutivo nacional con diferentes modalidades de administración y control. Por un lado, RTA contenía a Canal 7 y a la red de 49 emisoras de Radio Nacional. Por otra parte, del Ministerio de Educación dependían las señales Encuentro, Pakapaka y DeporTV. A estos se sumaban TecnópolisTV, dependiente del Ministerio de Ciencia, Tecnología e Innovación productiva, e IncaaTV, vinculado al Instituto Nacional de Cine y Artes Audiovisuales (INCAA). A estas señales deben agregarse Acua Mayor y Acua Federal, iniciativas producidas en el marco de la Planificación Estratégica del Sistema Argentino de Televisión Digital Terrestre coordinado por un Consejo Asesor creado por el decreto 1148/2009 y dependiente del extinto Ministerio de Planificación Federal, Inversión Pública y Servicios.

La legislación vigente al 10 de diciembre de 2015 ubicaba a RTA bajo el ala institucional de la Secretaría de Comunicación Pública de la Jefatura de Gabinete de Ministros, que tenía como uno de sus objetivos "supervisar" su funcionamiento.

Se desprendía de la Ley 26522 de Servicios de Comunicación Audiovisual (LSCA) que cinco instancias institucionales diferentes, creadas por la misma norma, podían ejercer supervisión de los medios gestionados por el Estado nacional. En forma específica, la Comisión Bicameral de Promoción y Seguimiento de la Comunicación Audiovisual y el Consejo Consultivo Honorario de los Medios Públicos debían controlar e informar sobre el funcionamiento de estos medios. En forma más general, el Consejo Federal de Comunicación Audiovisual (COFECA), la Autoridad Federal de Servicios de Comunicación Audiovisual (AFSCA) y la Defensoría del Público de los Servicios de Comunicación Audiovisual debían velar por el cumplimiento de la norma, preparar informes y retomar denuncias.

La Ley 26522 plantea diferentes espacios para distintas instancias de representación social y política, con la potencialidad de vehiculizar controles, exigencias y/o reclamos. De este modo, la Comisión Bicameral, compuesta por ocho senadores y ocho diputados, tiene potestad de nombrar a tres directores en RTA en representación de las tres primeras minorías parlamentarias. En la conformación del directorio también participó el COFECA, que elegía a dos directores, mientras el Ejecutivo nacional tiene la potestad de nombrar al presidente y al director restante. Cada director tiene un mandato de cuatro años con la posibilidad de una sola reelección. Con todo esto, la norma contempla un directorio plural y representativo de fuerzas políticas, gremiales y académicas, diferenciado de la conducción ejecutiva de los medios abarcados por RTA. 
Un contrapeso importante a la designación directa a cargo del PEN del presidente del organismo es la obligación de mantener una diferencia de dos años entre "el inicio del mandato de los directores y del Poder Ejecutivo".

El Decreto de Necesidad y Urgencia 267/15 dictado por el gobierno de Cambiemos a poco de su asunción disolvió el AFSCA y el COFECA, a los que sustituyó por el Ente Nacional de Comunicaciones (Enacom) y el Consejo Federal de Comunicaciones (COFECO) respectivamente. Los nuevos entes creados mantuvieron las funciones de control del cumplimiento de la Ley 26522.

Con los decretos 12, 13 y 26 del primer día de gobierno de Cambiemos se creó el SFMCP en la órbita de la Jefatura de Gabinete de Ministros, con rango y jerarquía de ministro para su titular nombrado, Hernán Lombardi. Entre sus funciones se señalaba: "intervenir en la administración y el funcionamiento" de RTA, coordinar con el Ministerio de Educación "el contenido y funcionamiento" de Encuentro, Paka Paka, Depor TV y del Polo de Producción Audiovisual, así como "entender" en la operación del Banco Audiovisual de Contenidos Universales Argentino (BACUA).

Se subraya aquí la atribución formal del SFMCP para "intervenir en la administración" de RTA. Asimismo, se ordenaba transferir a su órbita la agencia de noticias estatal Télam.

Por el decreto $237 / 15$ en el SFMCP se crearon estructuras diferenciadas para atender a cuestiones específicas: por un lado la Secretaria de Contenidos Públicos se hizo responsable de los contenidos producidos en el marco del Consejo Asesor del Sistema Argentino de la Televisión Digital Terrestre (1), además de la articulación con el Ministerio de Educación en la generación de contenidos para las señales Encuentro, DeporTV y Paka Paka; por el otro, la Secretaria de Medios Públicos tiene los objetivos de "asistir en la administración y funcionamiento" de RTA y de Télam.

Con el cambio de gobierno renunciaron los directores de Radio Nacional y Canal 7, además del presidente de RTA en funciones desde 2008, a lo que se sumó luego la remoción del director de la junta de gobierno que representaba al Ejecutivo saliente. Estos movimientos dan cuenta de la apertura de un nuevo momento institucional, más allá de la continuidad del directorio.

El Ejecutivo cubrió los cargos vacantes con nuevas designaciones que colocaron a un nuevo presidente del directorio de RTA. En los lugares restantes del organismo se mantuvieron los representantes que ejercían en ese momento su actividad según la normativa vigente (2).

Por otra parte, en abril de 2017 el presidente de RTA firmó una resolución para despedir a 21 trabajadores de igual cantidad de emisoras de la red federal de Radio Nacional. Los despedidos se habían desempeñado como directores de sus emisoras durante la gestión 
precedente pero dejaron esos lugares para cumplir otras tareas a partir de la llegada de las nuevas autoridades.

Entre otros aspectos institucionales se cuenta el cambio de dinámica en el funcionamiento del directorio de RTA. Durante 2016 prácticamente no se produjeron reuniones formales mensuales con participación de todos los directores para el análisis y aprobación de propuestas. Según explicaron los directores Verano y Cantariño (3), este mecanismo asentado con una periodicidad y formalidad bien estructurada hasta el cambio de gobierno (de Charras, 2016) dejó lugar a un funcionamiento en base a encuentros informales y reuniones fuera de agenda entre la presidencia del organismo y los diferentes directores, en el marco de un vínculo "propositivo y amistoso". Los entrevistados concuerdan en considerar esta situación como producto de un periodo de transición y aprendizaje, generado por los cambios en el directorio.

La política de contrataciones en Canal 7 (o TV Pública) prefirió evitar las coproducciones de programas y las producciones independientes en favor de la contratación de conductores o periodistas concretos y la utilización del equipo de planta. La única erogación que debió ser autorizada por el directorio por el alto monto manejado fue el pago de derechos para la transmisión de la Copa América Centenario y los Juegos Olímpicos de Río de Janeiro.

En cuanto a la entrega de informes consignada por ley, a lo largo del periodo se presentó un informe al Consejo Consultivo Honorario pero no sucedió lo mismo con la Comisión Bicameral vinculada al área.

Los cambios institucionales producidos en RTA por el Ejecutivo nacional al inicio de 2016 generan dudas sobre la integración del directorio dado que su recambio debió producirse en diciembre de 2017, al menos de aquellos cinco integrantes que fueron ratificados por 4 años para un segundo y último mandato en diciembre de 2013. Estos continuaban en la nómina en septiembre de 2018, salvo el caso de Cantariño, designado por el COFECA, quien renunció a su cargo en enero de ese año. En el caso de las designaciones del Ejecutivo en el directorio se ha quebrado el principio de desacople sancionado por ley respecto al periodo de gobierno nacional.

Un problema normativo a resolver es que el COFECA tenía a su cargo la propuesta de dos directores de la empresa estatal, pero esta función no fue trasladada al COFECO, creado por el DNU 267/15 para sustituir al disuelto consejo. Además, para septiembre de 2018 el mencionado COFECO no se había constituido todavía y la Comisión Bicameral del Congreso nacional, que debe proponer a tres nuevos integrantes del directorio, retomó en el último mes analizado su trabajo luego de una prolongada inactividad. Sin embargo, no abordó este tema. 
Adicionalmente, el decreto 1052/17 estableció que las tareas de los directores de RTA se realizarán ad-honorem a partir de las próximas designaciones.

\section{Laberintos normativos y decisiones gubernamentales}

Al 30 de septiembre de 2018 todavía no se había constituido el mencionado COFECO. Como consecuencia de esto, desde 2017 no se pudo cumplir con los artículos 77, 78 y 79 de la LSCA, que garantizaban el acceso universal a contenidos de interés relevante. Fue así porque, según el artículo 77, una de las funciones del disuelto COFECA era elaborar un listado anual de acontecimientos de interés general "respecto de los cuales el ejercicio de derechos exclusivos deberá ser justo, razonable y no discriminatorio". Particularmente entre 2010 y 1015 , pero también durante 2016, y gracias a los listados de eventos de interés público elaborados por el COFECA, la TV Pública utilizaba esta norma para adquirir los derechos de emisión de encuentros deportivos de gran convocatoria, incluso cuando otros canales los emitían.

Según el DNU 267/15, mientras el COFECO no se constituyese, sus tareas debía cumplirlas el Ministerio de Comunicaciones. Sin embargo, el decreto 513/17 trasladó al Ministerio de Modernización la responsabilidad de conformar ese espacio ante la disolución del Ministerio de Comunicaciones. La cartera de Modernización no avanzó con este espacio pero emitió la resolución 703-E/17 con la que creó unilateralmente una lista de eventos de interés público que incluyó un sólo ítem: todos los partidos oficiales de la selección nacional de fútbol. Esto permitió la transmisión de la Copa Mundial de Fútbol 2018 en la TV Pública, pero impidió desde 2017 incorporar emisiones de competencias internacionales de otras disciplinas como boxeo, hockey, básquet o rugby. Así, lo que era un instrumento de participación ciudadana para favorecer el acceso a contenidos audiovisuales de trascendencia nacional derivó en una decisión unilateral para limitar un derecho garantizado legalmente.

Otra decisión del Ejecutivo nacional con fuerte incidencia en la televisión estatal fue la renegociación del programa Fútbol Para Todos en 2016 (4). A partir de esto, Canal 7 dejó de emitir los principales encuentros de la primera división del fútbol de Argentina. Estas transmisiones fueron negociadas con los tres principales canales comerciales de la Área Metropolitana de la Ciudad de Buenos Aires (Canal 13, Canal 2 y Canal 11). Esto significó desde 2016 para la emisora estatal la pérdida de un contenido convocante. Esta modalidad de televisación se extendió hasta agosto de 2017, cuando el fútbol profesional del país pasó a ser transmitido en su totalidad por dos señales comerciales. El acceso a estos encuentros requirió desde ese momento el pago de un abono complementario al servicio de TV paga. 
Esta decisión significó también el retorno a una lógica de subsidiariedad en la función de los medios en manos del Estado.

\section{Una nueva concepción para las señales temáticas}

Por medio del decreto 1222 del 5 de diciembre de 2016, el diseño institucional sufrió una nueva modificación con la creación de Contenidos Públicos Sociedad del Estado en jurisdicción del SFMCP. Esta sociedad tiene por objeto "la gestión, operación, desarrollo y explotación de las señales Encuentro, Paka Paka y Depor TV, Acua Mayor y Acua Federal (...) así como la administración del polo de producción audiovisual y del acervo del Banco Audiovisual de Contenidos Universales Argentinos (BACUA)". La nueva sociedad está dirigida por tres directores nombrados por el Gobierno nacional.

El mismo decreto crea el estatuto social de Contenidos Públicos Sociedad del Estado y en su artículo 7 transfiere el presupuesto, personal, inmuebles, así como funciones, marcas, registros, patentes y demás bienes inmateriales concernientes y/o vinculados a Acua Mayor y Acua Federal y al BACUA. Este artículo tiene la particularidad de transferir un inmueble que no existe, ya que el funcionamiento del BACUA y de las señales Acua Federal y Acua mayor, todos sus equipos, tendidos y archivos funcionaban en un subsuelo prestado por el Ministerio de Defensa que aloja a la empresa Fabricaciones Militares en la Ciudad de Buenos Aires.

Desde diversos sectores, sobre todo desde los sindicatos de educación, se denunció que este decreto ignora y vulnera los artículos 101, 102 y 142 de la Ley 26.206 de Educación Nacional.

El Decreto 1.222 desplaza a Encuentro, Paka Paka y DeporTV de la jurisdicción de la sociedad Educ.ar, dependiente del Ministerio de Educación. Eso supone un cambio de perspectiva en la generación de contenidos para esas señales y descalza al menos en una vinculación institucional determinada por Ley su función educativa y articulada con plataformas digitales y programas de educación formal.

Este reconocimiento no supuso una inyección de relevancia en las nuevas señales estatales, más bien todo lo contrario. En enero de 2017 se quitaron del sistema digital terrestre las señales Acua Federal y Acua Mayor. Junto con esta decisión, se dio de baja también la página on demand de series producidas por el Estado Nacional: cda.gob.ar.

Finalmente, el último día de 2017 se anunció el despido de todas las personas que se desempeñaban en el subsuelo del Ministerio de Defensa. La paradoja que comenzaba en el decreto que mencionaba un mobiliario que no existía terminó con el despido de 160 trabajadores con el argumento de la falta de potestad sobre el espacio de Fabricaciones Militares. 
Más de un año después de dar de baja el portal cda.gob.ar, el SFMCP lanzó en mayo de 2018 la plataforma digital Cont.ar. De acceso gratuito como su predecesora, esta página aloja las producciones audiovisuales y radiofónicas producidas y financiadas por el Estado nacional. Allí se ofrecen contenidos on demand pero también en directo con el sistema de streaming, que pueden reproducirse en computadoras, tabletas y teléfonos móviles. Este espacio recupera la producción nacional producida por el Estado nacional que alojaba cda.gob.ar.

Finalmente, la Resolución 4694 de julio de 2018 del Enacom resolvió que la inclusión de las señales temáticas de Contenidos Públicos S. E. en servicios de televisión paga "quedará sujeta a las condiciones convenidas entre las partes", y destaca que su "puesta a disposición en forma gratuita (...) generará la obligación de su inclusión”. Tanto en las resoluciones 1394/16 y 4337/18 como el artículo 65 de la LSCA, especifican que los servicios de televisión por suscripción por cable y satelitales tiene la obligación de incluir en su grilla las señales del Estado nacional. La resolución incluye un condicionamiento que tiende a relativizar esta obligación pues la supedita a su ofrecimiento gratuito por parte de la empresa productora.

\section{Definiciones sobre el financiamiento de los medios del Estado nacional}

A lo largo de 2016, los directores del SFMCP y RTA, así como los gerentes de Canal 7 y Radio Nacional, presentaron como valores positivos de sus gestiones el ahorro en sus presupuestos. Esto también fue ponderado en el informe de gestión presentado por RTA al Consejo Consultivo Honorario de los Medios Públicos con el argumento de la búsqueda de austeridad en el manejo de recursos. Se anunció la reducción en los montos de los contratos artísticos (periodistas contratados), la eliminación de coproducciones en la TV Pública, el ahorro de un $30 \%$ en el presupuesto de Radio Nacional y la reducción de profesionales para coberturas en el exterior.

En 2017 y 2018 se profundizó esa perspectiva y el propio presidente del SFMCP reconoció un plan de reducción de costos debido a que el presupuesto anual se mantendría en 2018 sin aumentos respecto de 2017 (Respighi, 2018). La inflación para los primeros nueve meses de 2018 era de 32,4\%, según la medición del Índice de Precios al Consumidor del Instituto Nacional de Estadísticas y Censos (INDEC).

En efecto, desde los últimos meses de 2017 el SFMCP impulsó la retracción salarial y la reducción de la planta de trabajadores en los medios públicos nacionales. Esta política encontró la resistencia de los trabajadores de Radio Nacional y particularmente de Canal 7 (TV Pública). Fue luego del despido de 14 personas de la emisora radial y del impulso de un programa de jubilaciones anticipadas desde RTA. 
Al mismo tiempo, el SFMCP propuso desde fines de 2017 en Canal 7 la modificación del convenio colectivo de prensa televisada y del convenio de empresa. Esta propuesta se transformó desde febrero de 2018 en una medida unilateral de RTA. Esto significó la eliminación de las horas extras y la reducción de hecho de los sueldos, que en algunos casos llega al 40\% del salario, según el Sindicato de Prensa de Buenos Aires (Sipreba). En este contexto, la empresa planteó una negociación paritaria sin aumentos salariales. El gremio contabilizaba alrededor de 200 trabajadores retirados a través de los planes de retiros voluntarios y de jubilaciones anticipadas desde diciembre de 2017, sin la sustitución de esos empleados.

Las consecuencias inmediatas fueron la reducción de 30 a 14 horas semanales de noticieros, con la pérdida de las emisiones de los fines de semana, y el inicio de medidas de fuerza con repercusiones en la pantalla del canal durante 2018. Esta política de reducción de costos disminuyó también la salida de móviles para transmisiones desde exteriores así como las coberturas periodísticas con enviados a acontecimientos internacionales.

Según el criterio oficial, la planta se encontraba sobredimensionada, con superposición de áreas e "irregularidades" (SFMCP, 2017) no especificadas.

Por otra parte, la retirada del Estado nacional en el financiamiento de la ficción nacional desde 2016 también afectó a la pantalla de la TV Pública y otras señales estatales. Es así porque entre 2010 y 2015 la emisora puso al aire series surgidas del Plan Operativo de Fomento de la Televisión Digital. El protagonismo de este género lo constata Carboni cuando señala que entre 2002 y 2015 circularon por la emisora estatal 66 programas de ficción, y de estos casi $70 \%$ se emitió en la etapa 2011-2015 (5). En términos generales, durante el periodo 2011/2015, la política del Plan operativo llevó adelante 43 concursos federales que significaron más de 3000 horas de contenido audiovisual alojado en el BACUA. Durante el período 2016/2017 se llevaron adelante tres concursos que significaron 24 horas de contenido al BACUA (6).

\section{Las cifras del financiamiento en RTA}

Resulta desafiante establecer una medida justa, ideal o suficiente para el financiamiento de los medios públicos. Aún así, es posible observar tendencias y realizar comparaciones entre cifras que ofrezcan elementos válidos para interpretaciones. En este caso, el análisis se focaliza en RTA. 


\begin{tabular}{c|c|c|c|c|c|c|c|} 
Año & $\begin{array}{c}\text { Presupuesto } \\
\text { nacional }\end{array}$ & $\begin{array}{c}\text { Publicidad } \\
\text { oficial }\end{array}$ & $\begin{array}{c}\text { Publicidad } \\
\text { comercial }\end{array}$ & $\begin{array}{c}\text { Ley } 26.522 \\
\text { Art.97 }\end{array}$ & $\begin{array}{c}\text { Recursos } \\
\text { totales }\end{array}$ & $\begin{array}{c}\text { Suba } \\
\text { interanual }\end{array}$ & IPC \\
\hline $\mathbf{2 0 1 6}$ & 2.495 .841 .212 & $\begin{array}{c}33.153 .138 \mathrm{C} 7 \\
6.154 .256 \mathrm{RN}\end{array}$ & $\begin{array}{c}237.835 .142 \mathrm{C} 7 \\
1.259 .201 \mathrm{RN}\end{array}$ & 377.545 .657 & 3.319 .651 .516 & $28,30 \%$ & $41 \%$ \\
\hline$\%$ & $79,18 \%$ & $1,24 \%$ & 7,58 & $11,97 \%$ & $100 \%$ & & \\
\hline $\mathbf{2 0 1 7}$ & 2.581 .085 .000 & $\begin{array}{c}24.493 .985 \mathrm{C} 7 \\
6.386 .922 \mathrm{RN}\end{array}$ & $\begin{array}{c}210.179 .523 \mathrm{C} 7 \\
1.643 .376 \mathrm{RN}\end{array}$ & 495.862 .710 & 3.319 .651 .516 & $5,00 \%$ & $26,10 \%$ \\
\hline$\%$ & $77,70 \%$ & 0,93 & $6,38 \%$ & $14,90 \%$ & $100 \%$ & & \\
\hline $\mathbf{2 0 1 8}$ & 2.500 .000 .000 & & & & & - & $35 \%$ \\
\hline
\end{tabular}

Tabla 1. Financiamiento RTA durante la Alianza Cambiemos (Linares, 2018) (7).

Si bien se observa un crecimiento de los montos totales destinados a Canal 7 y Radio Nacional en cada año, cabe prestar mayor atención a los porcentajes de los aumentos interanuales y su relación con el aumento en el costo de vida. Esto permite construir tendencias más demostrativas de la magnitud real de las variaciones. Así, los aumentos registrados quedan por debajo de las cifras de inflación.

La variación interanual promedio del presupuesto de RTA en la etapa 2016-2017 fue de 16,6\% mientras la inflación promedio fue de $33 \%$, sin tomar las cifras previstas para 2018 , en la que no se prevén aumentos del presupuesto mientras la devaluación de la moneda experimentada entre abril y septiembre habilitaba cálculos de inflación de entre 35 y $50 \%$. Así, el periodo de Cambiemos marca una derrota de los aumentos presupuestarios contra la inflación por 16 puntos porcentuales (que pueden extenderse a 23 puntos de cumplirse las previsiones presupuestarias de la propia gestión del SFMCP para 2018 frente a al escenario más favorable de inflación anual).

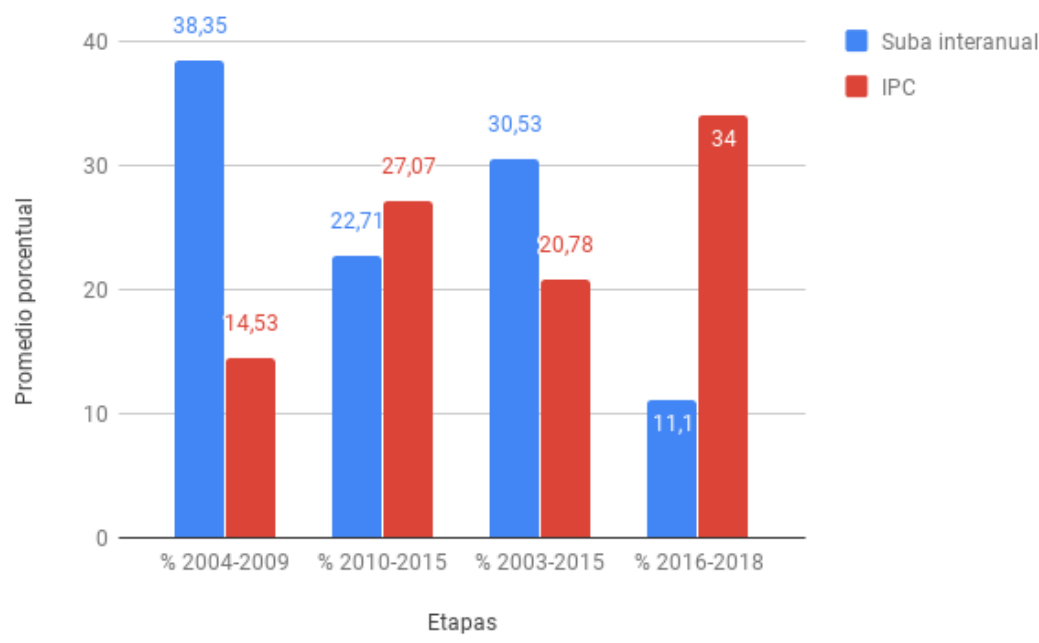

Gráfico 1. Promedios de aumentos anuales e inflación anual. Comparativo por etapas (Linares, 2018). 
Otra opción para evaluar el financiamiento público hacia los medios en cuestión consiste en medir su presupuesto de acuerdo a la población residente en el país. Para esto se deben dividir los montos asignados a los medios por la cantidad de habitantes. Con estos parámetros, en Argentina el presupuesto por habitante para RTA fue en 2014 de 5,3 dólares estadounidenses mientras en 2015 fue de 5,5 dólares por habitante. Según esta medición, el financiamiento público bajó, en 2016, a 4,7 dólares por habitante y más aún en 2017: 4,3 dólares.

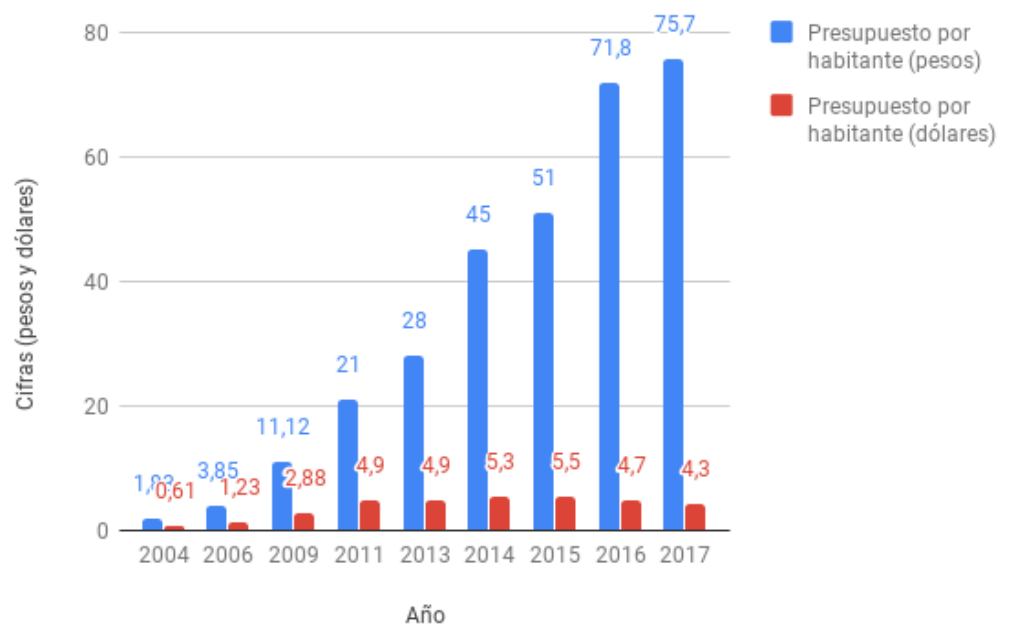

Gráfico 2. Evolución del presupuesto de RTA por habitantes. Periodo 2004-2017 (Linares, 2018) (8).

Si a esto se suman las partidas del Presupuesto Nacional destinadas al SFMCP para 2017 (2.476.906.920 pesos), el monto llega 335.061.181 dólares (5.796.558.436 pesos), lo que equivale a 7,6 dólares por habitante o (132 pesos).

Esta cifras se mantenían alejadas del promedio del presupuesto por habitante medido entre 18 países desarrollados, que era de 78 dólares estadounidenses en 2009, con extremos de 164 dólares en Noruega y 3,5 dólares en Estados Unidos. El mismo promedio en 2011, que sólo toma los recursos públicos provenientes del canon de servicio público o del presupuesto estatal (deja afuera ingresos comerciales), era de 85 dólares por habitante (Nordcity, 2011 y 2013).

Se debe considerar que los presupuestos evaluados sólo incluyen a los medios integrantes de RTA y no al resto de los medios del Estado nacional creados en la última década. Además, no todas las empresas estatales tienen la misma cantidad de medios y trabajadores a cargo, ni la misma modalidad de financiamiento. En el caso de RTA, el grueso del financiamiento llega de los recursos presupuestarios ( $77 \%$ en 2017 , según los números oficiales de la empresa). Estos fondos son definidos por el Poder Ejecutivo y aprobados por el Congreso nacional. 
Los montos medidos en dólares estadounidenses deben analizarse con la precaución de considerar las variaciones en los tipos de cambio y en el valor de las monedas locales. Por tanto, esta comparación debe abordarse como portadora de referencias para la reflexión más que como un cuadro de relaciones directas entre las cifras. En todo caso, los números recogidos dan cuenta de una caída del presupuesto en dólares en función de la devaluación de la moneda nacional frente al dólar estadounidense.

\section{El federalismo sui generis en Radio Nacional}

La principal apuesta concretada por la gerencia de Radio Nacional en 2017 consiste en transmitir una misma programación en simultáneo en sus 49 emisoras. Debido a que cada estación cuenta con una señal en AM y otra en FM (salvo en el caso de la ciudad capital, donde hay tres FM), la dirección de Radio Nacional instó a las emisoras a mover sus programaciones locales a su banda en AM para dejar en la FM la programación unificada con diseño centralizado, algo que no ocurría anteriormente.

La propuesta reproduce un federalismo sui generis pues se trata de una grilla compuesta casi en su totalidad por programas generados en la Ciudad de Buenos Aires. Esta programación se decidió desde la dirección de cabecera de Radio Nacional. Esto es, el espacio de FM destinado legalmente a cada estación se nutre de un contenido diseñado en la gerencia central donde de lunes a viernes el aporte federal lo hacen emisiones en horarios marginales.

A esta propuesta puede sumarse la decisión de incluir en la programación de $A M 870$ de la Ciudad de Buenos Aires en el horario de 22:00 a 00:00 horas una emisión en dúplex con la $F M$ Nacional Rock (señal temática en FM también ubicada en la capital argentina). Esto genera una disminución de contenidos emitidos para un territorio densamente poblado, lo que deriva en una pérdida en términos de diversidad y pluralismo.

\section{Conclusiones}

Desde el inicio del periodo se evidenció la intervención decidida del Ejecutivo nacional en los medios estatales. El decreto fue el mecanismo elegido para operar los cambios, lo que supuso en algunos casos desatender la normativa vigente y en otros eludir el debate en torno a estas decisiones. 
Las modificaciones normativas implementadas asignan una mayor capacidad formal de intervención del Ejecutivo nacional en RTA. De la posibilidad de "supervisar" su funcionamiento que tenía la Secretaría de Comunicación Pública hasta 2015 se pasó a la potestad del SFMCP de "intervenir" en su administración. Esto coloca en entredicho la autonomía de la empresa estatal.

El quiebre parcial de la institucionalidad de RTA y las designaciones en su directorio condicionan la futura conformación del directorio de RTA. Se trata de una instancia colegiada de dirección que fue debilitada y que constituye un eje fundamental de la arquitectura institucional ofrecida por la vigente Ley 26522 a la empresa estatal.

Independientemente de la voluntad de unificar la administración de los medios estatales en un mismo organismo, la creación de Contenidos Públicos S. E. en la órbita del SFMCP marca un cambio de rumbo para las señales consideradas hasta 2016 como educativas y como parte de un dispositivo articulado de propuestas pedagógicas.

La singular concepción de federalismo asumida por Radio Nacional tiende a replicar desde la red estatal el centralismo en la producción radiofónica extendido en los medios comerciales, lo que redunda en la pérdida de diversidad, pluralismo y expresiones locales.

El enfoque de financiamiento y la reducción real de los recursos destinados a RTA en los presupuestos de 2016, 2017 y 2018 generan interrogantes sobre el rol proyectado para estos medios, sobre su capacidad de ofrecer contenidos de interés público y sobre su posibilidad de asumir un espacio protagonista en el escenario mediático. Los aportes públicos representan casi $80 \%$ de los ingresos de RTA, por lo que su rebaja exige compensaciones importantes desde otros ingresos (como la publicidad comercial), difícil de imaginar en el corto plazo.

La política de reducción de costos generó la pérdida de puestos laborales y del poder adquisitivo de sus trabajadores. Detrás de objetivos de reorganización, la gestión del SFMCP debilitó la capacidad de producción propia de estos medios. Así, se avanzó sobre la dotación de personal y sus salarios en nombre de la eficiencia operativa, pero se limitó la incidencia de estos proyectos audiovisuales. En las definiciones sobre su sostenimiento no sólo se ponen en juego puestos laborales, sino la capacidad de brindar contenidos valorados por la audiencia con la función social que debe ofrecer un medio público.

La renegociación y posterior finalización del programa Fútbol para Todos repercutió negativamente en los niveles de audiencia de Canal 7 y lo colocó en una lógica subsidiaria en el sistema mediático, además de condicionar al público a la suscripción de sistemas de televisión pagos para acceder a este contenido.

Estas decisiones en términos institucionales, presupuestarios y de contenido no pueden considerarse desvinculadas de la fuerte caída desde 2016 en los niveles de audiencia de Radio 
Nacional y la TV Pública (9). Pese a carecer de fiabilidad comprobada en Argentina, la mediciones de audiencia permiten elaborar comparaciones. A partir de estas, se observa en estos medios una caída de al menos $50 \%$ de la audiencia respecto a 2015 . Si bien las obligaciones de estos medios no tienen como prioridad el logro de altos números de rating, entre sus misiones ocupa un lugar importante el vínculo estable con diferentes audiencias.

Durante 2017 la tendencia continuó a la baja, con números que alertan fuertemente sobre la función social del medio público cuando no tiene la capacidad de interpelar a la ciudadanía. La falta de audiencia, junto con la retirada de la producción y el fomento al contenido propio, consolida un modelo de televisión pública lejos de su función social y de la concepción del derecho a la comunicación, lo que redunda en ciudadanías más débiles y desamparadas frente a un campo cada vez más dominado por los jugadores privados. Este trabajo evidencia un cambio de perspectiva en la gestión de los medios estatales nacionales desde 2016. En esta tendencia, las emisoras se desplazan de un rol complementario y protagonista a otro subsidiario y marginal dentro del sistema mediático.

\section{Notas}

(1) Se incluyen aquí las producciones de los canales Acua Mayor y Acua Federal así como el trabajo vinculado a Contenidos Digitales Abiertos (CDA) y el Banco Audiovisual de Contenidos Universales Argentino (BACUA).

(2) La composición la completan: Néstor Cantariño, designado por el COFECA; Alejandro Verano, también designado por el COFECA; María Lenz, designada por la primera minoría parlamentaria (Frente para la Victoria); José César Gustavo Cusinato, designado por la segunda minoría parlamentaria (Unión Cívica Radical); y Marcelo Adolfo Romeu, designado por la tercera minoría parlamentaria (Partido Socialista).

(3) Entrevistas personales con Néstor Cantariño en la Ciudad de Buenos Aires el 3 de noviembre de 2016 y con Alejandro Verano el 22 de noviembre de 2016.

(4) Fútbol para Todos fue el programa de política pública liderado por la Jefatura de Gabinete de Ministros, surgida del acuerdo firmado en agosto de 2009 entre el Ejecutivo nacional y la Asociación del Fútbol Argentino. Este proyecto adquirió los derechos de televisación de los partidos del fútbol argentino de primera división y afrontó la inversión en la producción de las emisiones. Si bien muchos partidos fueron difundidos por otros canales de aire y cable, la pantalla de Canal 7 fue la principal boca de difusión de los encuentros.

(5) Ver Carboni, Ornela. ¿La ficción televisiva argentina en descenso? Síntesis de las ficciones argentinas producidas y emitidas entre 2002 y 2015. Recuperado de https://bit.ly/2VhThmQ

(6) Uno de ellos era de financiación mixta y todos fueron asociados a pantallas de primera emisión, entrando en la posibilidad de circulación del BACUA a partir de su segunda emisión.

(7) Todas las cifras están expresadas en pesos argentinos. Para 2018 se proyectan los recursos provenientes del Presupuesto Nacional previstos por el SFMCP y un escenario favorable de inflación anual según las previsiones del Ejecutivo nacional. 
(8) El paso de las cifras en pesos a dólares se realizó con el tipo de cambio oficial calculado el 9 de septiembre de cada año. La fecha fue elegida al azar. Los datos de población para cada año corresponden a las estimaciones entregadas por el Banco Mundial. Disponible en http://datos.bancomundial.org/pais/argentina?view=chat. Para 2017 se toman los números poblacionales de 2016 ante la ausencia de proyecciones.

(9) El rating promedio de 2016 de Canal 7 fue de 1.5. Esto es casi la mitad del promedio de 2015, que fue de 2.8, según los informes anuales de la única medidora de audiencias de Argentina: Ibope.

\section{Bibliografía}

Becerra, M. (2015). De la concentración a la convergencia. Política de medios en Argentina y América Latina. Buenos Aires: Paidós.

Becerra, M. y Mastrini, G. (2016). Políticas de medios del kirchnerismo. Análisis de las políticas de comunicación 2003-2015 y agenda pendiente. ANÁLISIS, 13, Friederich Ebert Stiftung-Argentina.

Becerra, M. y Mastrini, G. (2017). La concentración infocomunicacional en América Latina (2003-2015). Nuevos medios y tecnologías, menos actores. Universidad Nacional de Quilmes/Observacom: Bernal/Montevideo.

de Charras, D. (comp.). (2016). Implementación del sistema de indicadores de calidad de emisoras públicas para la evaluación de la Televisión Pública Argentina. Buenos Aires: Facultad de Ciencias Sociales, UBA.

Fuenzalida, V. (2001). La televisión pública en América Latina. Reforma o privatización. Santiago: Fondo de Cultura Económica.

Linares, A. (2018). El financiamiento de los medios estatales en Argentina en la posconvertibilidad. Tendencias, evaluaciones y comparaciones. Ponencia presentada en las Jornadas Transformaciones de las Industrias Culturales en la Era Digital, Maestría de Industrias Culturales de la Universidad Nacional de Quilmes, octubre.

Loreti, D. y Lozano, L. (2014). El derecho a comunicar. Argentina: Siglo XXI Editores.

Mastrini, G. y Mestman, M. (1995). ¿Desregulación o rerregulación? De la derrota de las políticas a las políticas de la derrota. Recuperado de https://core.ac.uk/download/pdf/38811053.pdf

Monje, D. (2006). Apuntes para el estudio de Políticas de Radio y TV frente a procesos de integración regional. Caso MERCOSUR. UNIrevista, Vol. 1.

Nordicity Group Ltd. (October 2013). Analysis of Government Support for Public Broadcasting and other Culture in Canada. Recuperado de https://bit.ly/2BZTZfU 
Oszlak, O. y O'Donnell, G. (1984). Estado y políticas estatales en América Latina: hacia una estrategia de investigación. En Flores, G. (ed.). Administración pública. Perspectivas críticas (pp. 378-415). San José: ICAP.

Pasquali, A. (1991). ¿Qué es una radiodifusión de servicio público?. En El Orden Reina. Caracas: Monte Ávila.

Respighi, E. (17 de abril de 2018). No diría que es un plan de ajuste, sino de austeridad. Diario Página/12. Recuperado de https://bit.ly/2qFYUg2

Sistema Federal de Medios y Contenidos Públicos (SFMCP) (2017). La Televisión Digital Abierta (TDA) seguirá funcionando con normalidad. Recuperado de https://bit.ly/2Cc55jP

Sistema de Información Cultural de la Argentina (Sinca) (2018). Encuesta Nacional de Consumos Culturales 2017. Ministerio de Cultura de la Nación, Buenos Aires. 